\title{
Persistent postural-perceptual dizziness (PPPD): a common, characteristic and treatable cause of chronic dizziness
}

\author{
Stoyan Popkirov, ${ }^{1}$ Jeffrey P Staab, ${ }^{2}$ Jon Stone ${ }^{3}$
}

\begin{abstract}
${ }^{1}$ Department of Neurology, University Hospital Knappschaftskrankenhaus Bochum, Ruhr-University Bochum, Bochum, Germany ${ }^{2}$ Department of Psychiatry and Psychology and Otorhinolaryngology - Head and Neck Surgery, Mayo Clinic Minnesota, Rochester, Minnesota, USA

${ }^{3}$ Centre for Clinical Brain Sciences, University of Edinburgh, Western General Hospital, Edinburgh, UK
\end{abstract}

\section{Correspondence to}

Dr Stoyan Popkirov, Department of Neurology, University Hospital Knappschaftskrankenhaus Bochum, Ruhr-University Bochum, 44892 Bochum, Germany; popkirov@gmail.com

Accepted 15 October 2017 Published Online First 5 December 2017

\section{SLinked}

- http://dx.doi.org/10.1136/ practneurol-2017-001817

\section{CrossMark}

To cite: Popkirov S, Staab JP, Stone J. Pract Neurol 2018;18:5-13.

\begin{abstract}
Persistent postural-perceptual dizziness (PPPD) is a newly defined diagnostic syndrome that unifies key features of chronic subjective dizziness, phobic postural vertigo and related disorders. It describes a common chronic dysfunction of the vestibular system and brain that produces persistent dizziness, non-spinning vertigo and/ or unsteadiness. The disorder constitutes a long-term maladaptation to a neuro-otological, medical or psychological event that triggered vestibular symptoms, and is usefully considered within the spectrum of other functional neurological disorders. While diagnostic tests and conventional imaging usually remain negative, patients with PPPD present in a characteristic way that maps on to positive diagnostic criteria. Patients often develop secondary functional gait disorder, anxiety, avoidance behaviour and severe disability. Once recognised, PPPD can be managed with effective communication and tailored treatment strategies, including specialised physical therapy (vestibular rehabilitation), serotonergic medications and cognitive-behavioural therapy.
\end{abstract}

\section{INTRODUCTION}

Persistent postural-perceptual dizziness (PPPD) is a chronic functional disorder of the nervous system, characterised by non-spinning vertigo and perceived unsteadiness (see box 1 for diagnostic criteria $\left.^{1}\right)$. The symptoms are exacerbated when patients assume upright postures and in situations with complex or moving visual stimuli (figure 1). The most common provocations are benign circumstances such as standing, walking, looking at traffic or sitting in a busy restaurant, which may be perceived as noxious or threatening. Symptoms of PPPD may be alleviated transiently in moments of distraction and may flare fleetingly without apparent provocation. PPPD is precipitated by episodes of vertigo or unsteadiness of vestibular, neurological or psychiatric origin. These triggers appear to induce involuntary utilisation of high-demand postural control strategies and an over-reliance on visual stimuli for spatial orientation. An initial period of high anxiety and excessive vigilance about the acute physical symptoms appears to perpetuate these reflexive processes, which are then inadequately mollified by top-down interactions among cortical vestibular, visual and threat assessment networks. $^{2-4}$ Maladaptive cognitive-behavioural responses commonly add secondary psychological and functional morbidity, such as fear of falling, anxiety or depressive disorders, and functional gait abnormalities. However, PPPD persists independently of any lesional or structural disease.

Different aspects of the disorder can dominate the clinical presentation, such as the primary symptoms of dizziness, unsteadiness and hypersensitivity to self-motion or complex visual stimuli, or the secondary complications of phobic avoidance of provocative situations and functional gait abnormalities. This can lead patients to different medical specialties (otolaryngology, psychiatry, neurology). Historically, the varied presentations resulted in the definitions of various overlapping nosological predecessors of PPPD, such as phobic postural vertigo, space-motion discomfort, visual vertigo, chronic subjective dizziness, psychogenic gait disorder and others. ${ }^{35}$ Arguments for differentiation of these disorders remain valid, but PPPD has recently emerged as a unifying and diagnostically unambiguous 

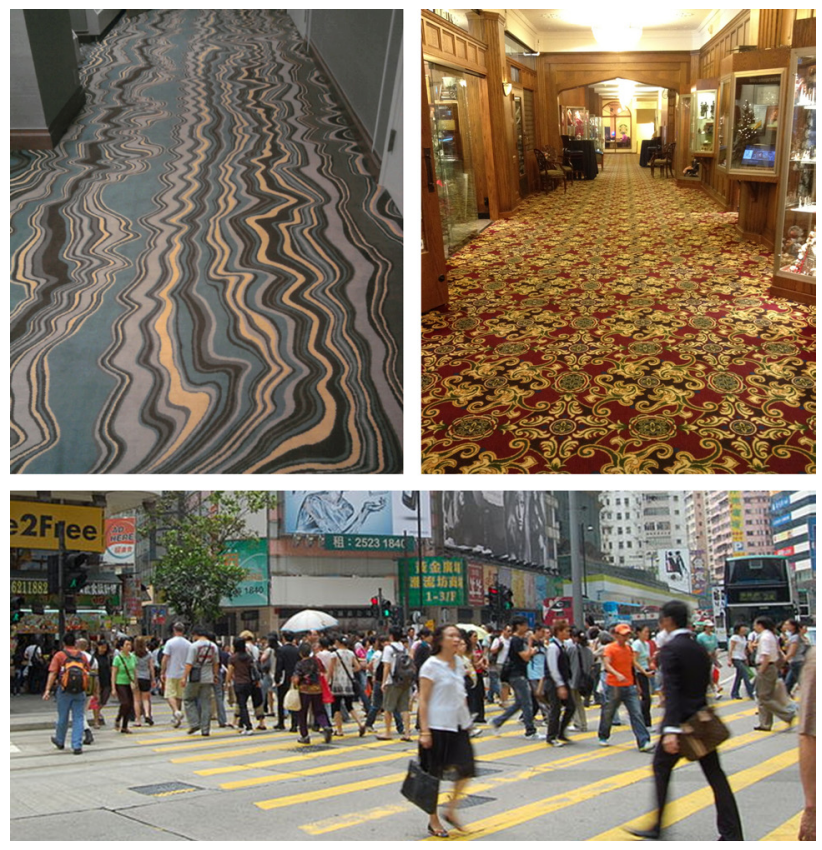

Figure 1 Complex visual stimulation such as patterned carpets (above) or busy streets (below). Above images are from the personal archive of Jeffrey P Staab. Photo below is Busy Street in Causeway Bay by edwin.11, licensed under the Creative Commons Attribution 2.0 Generic licence, cropped.

disorder that has been recognised by the WHO and will be included in the upcoming 11th edition of the International Classification of Diseases ${ }^{6}$ and the recently established International Classification of Vestibular Disorders. ${ }^{7}$ The new name, PPPD (best pronounced 'triple PD' or 'three PD' to avoid confusion with BPPV (benign paroxysmal positional vertigo)), aims to provide an aetiologically neutral but positive diagnostic term that avoids jumping to conclusions about whether the symptoms have arisen from a structural lesion or a purely 'phobic' or 'psychogenic' process.

This review will provide a neurobiologically informed clinical guide to recognising, diagnosing and treating PPPD as a common functional disorder in neurological practice.

\section{Epidemiology}

It is difficult to obtain validated data on incidence and prevalence for a recently redefined disorder that is seen by general practitioners, otolaryngologists, neurologists and psychiatrists. A UK population-based study of primary care found that $4 \%$ of all patients registered with a general practitioner experience persistent symptoms of dizziness, and most of those were incapacitated by their symptoms. ${ }^{8}$ In UK neurology outpatient clinics, $2 \%$ of all secondary referrals were diagnosed primarily with vertigo or dizziness, half of which were deemed psychological/ functional. ${ }^{9}$ In tertiary dizziness centres, either phobic postural vertigo or chronic subjective dizziness, two conceptual predecessors of PPPD, is the second most
Box 1 Bárány Society diagnostic criteria for persistent postural-perceptual dizziness ${ }^{1}$

A. One or more symptoms of dizziness, unsteadiness or non-spinning vertigo on most days for at least 3 months.

1. Symptoms last for prolonged (hours-long) periods of time, but may wax and wane in severity.

2. Symptoms need not be present continuously throughout the entire day.

B. Persistent symptoms occur without specific provocation, but are exacerbated by three factors: upright posture, active or passive motion without regard to direction or position, and exposure to moving visual stimuli or complex visual patterns.

C. The disorder is triggered by events that cause vertigo, unsteadiness, dizziness, or problems with balance, including acute, episodic or chronic vestibular syndromes, other neurological or medical illnesses, and psychological distress.

1. When triggered by an acute or episodic precipitant, symptoms settle into the pattern of criterion $\mathrm{A}$ as the precipitant resolves, but may occur intermittently at first, and then consolidate into a persistent course.

2. When triggered by a chronic precipitant, symptoms may develop slowly at first and worsen gradually.

D. Symptoms cause significant distress or functional impairment.

E. Symptoms are not better accounted for by another disease or disorder.

common diagnosis, accounting for about 15\%-20\% of all patient presentations. ${ }^{4}$ Prospective studies of patients who were followed for 3-12 months after acute or episodic vestibular ailments such as vestibular neuritis or BPPV suggest that persistent dizziness of the type seen in PPPD will develop in one out of four individuals, ${ }^{1}$ making the incidence of PPPD in this situation much higher than widely appreciated.

\section{DIAGNOSING PPPD}

Core symptoms and commonly associated clinical features The diagnosis of PPPD requires all Bárány Society criteria to be fulfilled. Thus, making the diagnosis relies on the patient history (Box $1^{1}$ ). Dizziness, unsteadiness and vertigo are notoriously difficult to describe and patients will offer various reports of symptoms, including non-spinning vertigo ("I feel a sense of motion - it's not whirling - even though I'm still"; "I feel as if my body is swaying like I'm on a boat"), unsteadiness ("I feel I'm about to fall"), light-headedness ("I feel as if I might pass out") and mild dissociation ("I feel spaced out/as if my legs are spongy/as if I'm floating”). Unsteadiness and non-spinning vertigo tend to dominate the clinical picture. Symptoms are exacerbated by upright posture, moving 
about actively or being moved passively (eg, standing, walking or riding in a vehicle), or being immersed in environments with complex or moving visual stimuli (eg, a hallway with complex patterned carpet, a supermarket aisle, looking at traffic). This visual hypersensitivity, which can occur in isolation as the symptom of 'visual vertigo', ${ }^{10}$ is a characteristic feature of PPPD, and often one of its most impairing features, especially in the modern world with its ever-growing intensity of visual stimulation.

Symptoms usually wax and wane (partially in accordance with introspection and distraction, alertness and exhaustion), but are generally described as persistent. Patients' account of symptom intensity, persistence and impairment in daily activities might appear at odds with their relatively benign appearance on casual observation in the clinic or testing in the vestibular laboratory. However, as in many functional neurological disorders, clinical 'inconsistencies' (eg, reports of persistent symptoms, but retained abilities to manage complex tasks intermittently when necessary) are a typical feature of attention-modulated disorders, and not signs of inauthenticity or malingering.

Patients will usually volunteer a triggering episode of vertigo or unsteadiness. In a quarter of cases the precipitating event is a peripheral or central vestibular disorder (eg, BPPV or vestibular neuritis), and in a further $20 \%$ of cases an attack of (vestibular) migraine can be identified. ${ }^{11}$ Panic attacks or generalised anxiety disorders account for $15 \%$ of triggers each. Trauma to the head associated with mild traumatic brain injury or whiplash injury is a trigger in up to $15 \%$, and autonomic disorders account for $7 \%$. Less common are dysrhythmias or adverse drug reactions (3\%). PPPD will usually develop as the immediate effects of the incipient event begin to fade.

Patients may give accounts of avoiding provocative situations with resultant impairment in social and occupational activities. Patients with PPPD may be quite consumed by their problem and present at their 'wits end'. They may describe anticipatory anxiety or incapacitation in situations where dizziness would be particularly problematic such as stairs or roads. When these secondary phobic thoughts and avoidance behaviours reach a distressing or disabling level, they warrant the additional diagnosis of a specific phobia such as fear of dizziness, fear of falling or agoraphobia. PPPD does not include recurrent falls or gait impairment, so a history of frequent falls or near falls or clinical signs of significant alterations in gait or stance merit consideration of gait diagnoses (see below).

PPPD is a diagnosis made by collecting a sufficient history to find that patients fulfil the diagnostic criteria for the disorder. It is not a diagnosis of exclusion. Physical examination, clinical laboratory testing, vestibular evaluation and diagnostic neuroimaging may be needed, not to 'rule out everything else', but to assess adequately the presence or absence of conditions in the differential diagnosis of PPPD. These include peripheral or central vestibular deficits, autonomic disorders, head injuries, or cardiac conditions. Historical enquiry should be extended to symptoms of migraine, panic attacks and generalised anxiety, the three conditions that most commonly coexist with PPPD. Side effects of medications from many classes can mimic some symptoms of PPPD. Consider temporal associations between symptoms of PPPD and initiation or changes in doses of prescription and non-prescription medications.

\section{Secondary psychiatric morbidity}

Screening for psychiatric morbidity may be accomplished easily using short, standardised, self-report questionnaires, such as the seven-item Generalised Anxiety Disorders Scale for anxiety disorders ${ }^{12}$ and the nine-item Patient Health Questionnaire for depression $^{13}$ (available free online at http://www. phqscreeners.com/). Fear of falling is more common among patients with PPPD than the conventional fear of panic attacks. A neurologist could usefully ask basic questions about whether the patient is avoiding situations where falls may occur. If fear of falling arouses excessive distress or avoidance behaviours, then intervention for this specific phobia is warranted.

\section{Secondary functional gait abnormality}

Most patients with PPPD as their only active diagnosis will perform routine tests of posture and gait normally or with minimal difficulty due to mild caution. Some patients, however, will show increased body sway and amplified compensatory movements of the arms during tests of stance (ie, Romberg test). Some patients may develop secondary features of a functional gait disorder with a slow or hesitant gait and/or a 'walking on ice' gait pattern. ${ }^{14}$ Unlike Parkinsonian freezing, this slowness remains unchanged after gait initiation. Distraction techniques during standing and walking can provide positive evidence that the unsteadiness has a broadly functional basis. ${ }^{15} 16$ During standing, this can be achieved by asking the patient to name a number written on their back or with a motor task such as using a phone or testing eye movements. ${ }^{17}{ }^{18}$ These are usually more effective than mental distraction such as serial sevens or listing months backwards. ${ }^{16}$ Counterintuitively, more demanding postural tasks, such as standing in tandem position or walking backwards, can also normalise body sway and gait. ${ }^{19}$ There may also be sudden knee buckling without falls; when present, this may have specific features of functional leg weakness such as a positive Hoover's sign or hip abductor sign. These examination 'tricks' provide a positive diagnosis of a functional gait disorder, and can be used to explain the diagnosis to patients, demonstrate the potential for reversibility and provide a starting point for therapy. ${ }^{201}$ 
Table 1 Related disorders and previous incarnations

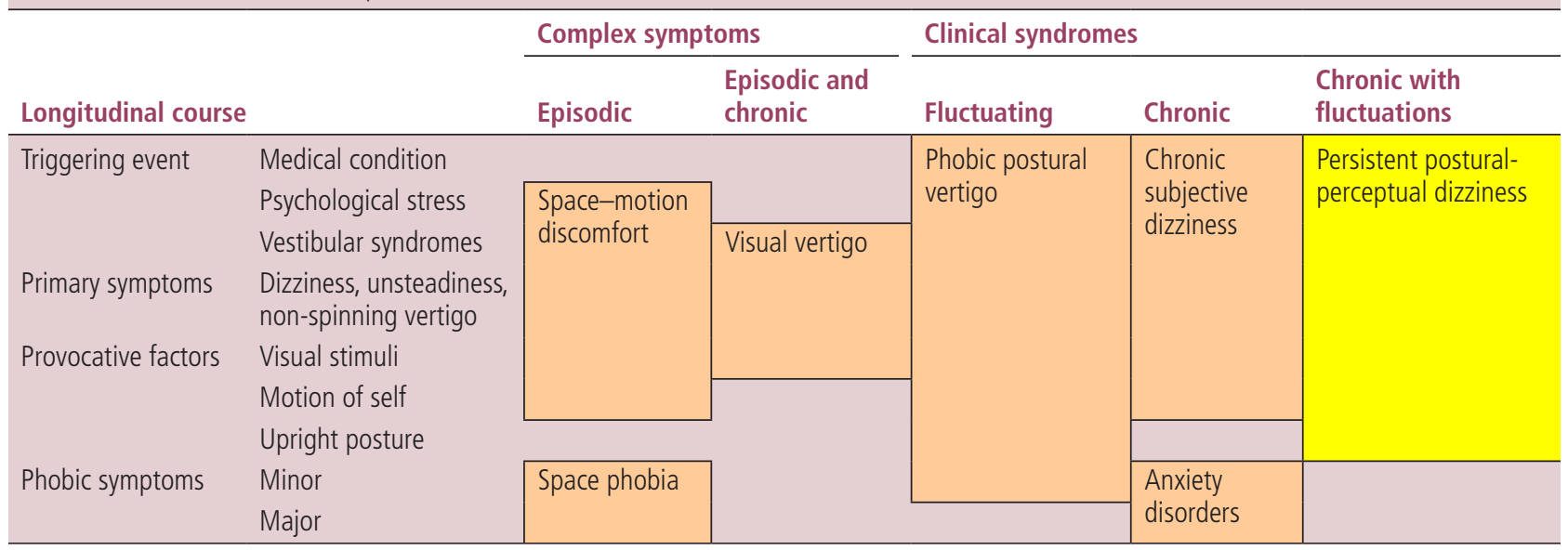

\section{Red flags in the differential diagnosis of PPPD}

Like other functional disorders of the nervous system, PPPD usually has a relatively acute onset, typically on the heels of its precipitating events, so an insidious symptom development should raise doubts about the diagnosis. In typical cases, patients do not experience symptom-free intervals, but rather a transition from acute to chronic symptoms (eg, acute spinning vertigo of vestibular neuritis to persistent unsteadiness of PPPD). In cases of episodic precipitants such as BPPV or vestibular migraine, symptoms of PPPD may remit and then return with recurrences of the triggering condition before settling into a persistent pattern. A more difficult diagnostic dilemma arises when the precipitating events themselves are insidious illnesses, such as degenerative disorders of the central nervous system or vestibular labyrinth. In these cases, clinicians may need to follow up patients prospectively with careful serial examinations to complete the diagnostic assessment.

Another possible pitfall is the co-occurrence of comorbid disorders. The presence of structural, metabolic or psychiatric disorders does not preclude the diagnosis of PPPD. On the contrary, PPPD can and does co-occur with many of the conditions in its differential diagnosis. For example, BPPV or vestibular migraine can trigger PPPD and then coexist with it, causing recurrent attacks of vertigo superimposed on the chronic symptoms of PPPD. Patients with Parkinson's disease and other structural gait disorders can develop functional dizziness. ${ }^{22}$ In this situation, PPPD is suggested most strongly by the presence of sensitivity to visually challenging stimuli when patients are seated. ${ }^{1}$ Panic disorder and generalised anxiety disorder may be causes or sequelae of PPPD. They may also predate the onset of PPPD without causing dizziness, but then worsen when PPPD is triggered by another condition. This circumstance is easily misconstrued as 'psychogenic dizziness', with resultant failure to properly identify all conditions contributing to the patient's morbidity. ${ }^{23}$ Hence, the clinician needs to consider carefully the time course of vestibular and associated symptoms to disentangle often interwoven aetiologies. The practical purpose of undertaking this task is to identify potentially reversible components of the presentation, which may be treatable through different therapies from those indicated for structural disorders or PPPD alone.

PPPD should not be used as a 'wastebasket' diagnosis for patients who present with non-specific or enigmatic dizziness without fulfilling all criteria for the diagnosis. In our clinical experience, these patients are more likely to have another problem, such as a slowly developing neurodegenerative disorder (eg, cerebellar degeneration) or evolving episodic disorder (eg, vestibular migraine). Prospective monitoring and reconsideration of the differential diagnosis is warranted for patients with insidious symptoms, short bouts of functional dizziness and multiple chronic complaints.

Finally, as with all functional disorders, it is a mistake to make a diagnosis of PPPD based on a history of childhood trauma or adulthood adversity (eg, physical or sexual abuse or neglect in childhood, relationship or employment struggles in adulthood). In a study of 546 patients with vestibular symptoms, rates of lifetime adversity were equally common among patients with structural and functional/psychiatric comorbidities. ${ }^{24}$

\section{Precursors of PPPD}

The history of this area is of evolving and overlapping clinically described syndromes (table 1). In 1986 Brandt and Dieterich first described stance-dependent and gait-dependent dizziness and unsteadiness as phobic postural vertigo. An obsessive-compulsive personality was included in the diagnostic criteria. ${ }^{25} \mathrm{Jacob}$ and colleagues $^{26}$ described space-motion discomfort as a clustering of uneasiness about spatial orientation and increased awareness of motion stimuli in some patients with dizziness in an anxiety clinic. In 1995 Bronstein $^{10}$ described visual vertigo, which saw patients who had otherwise recovered from acute vestibular disorders reporting dizziness when confronted with complex or 


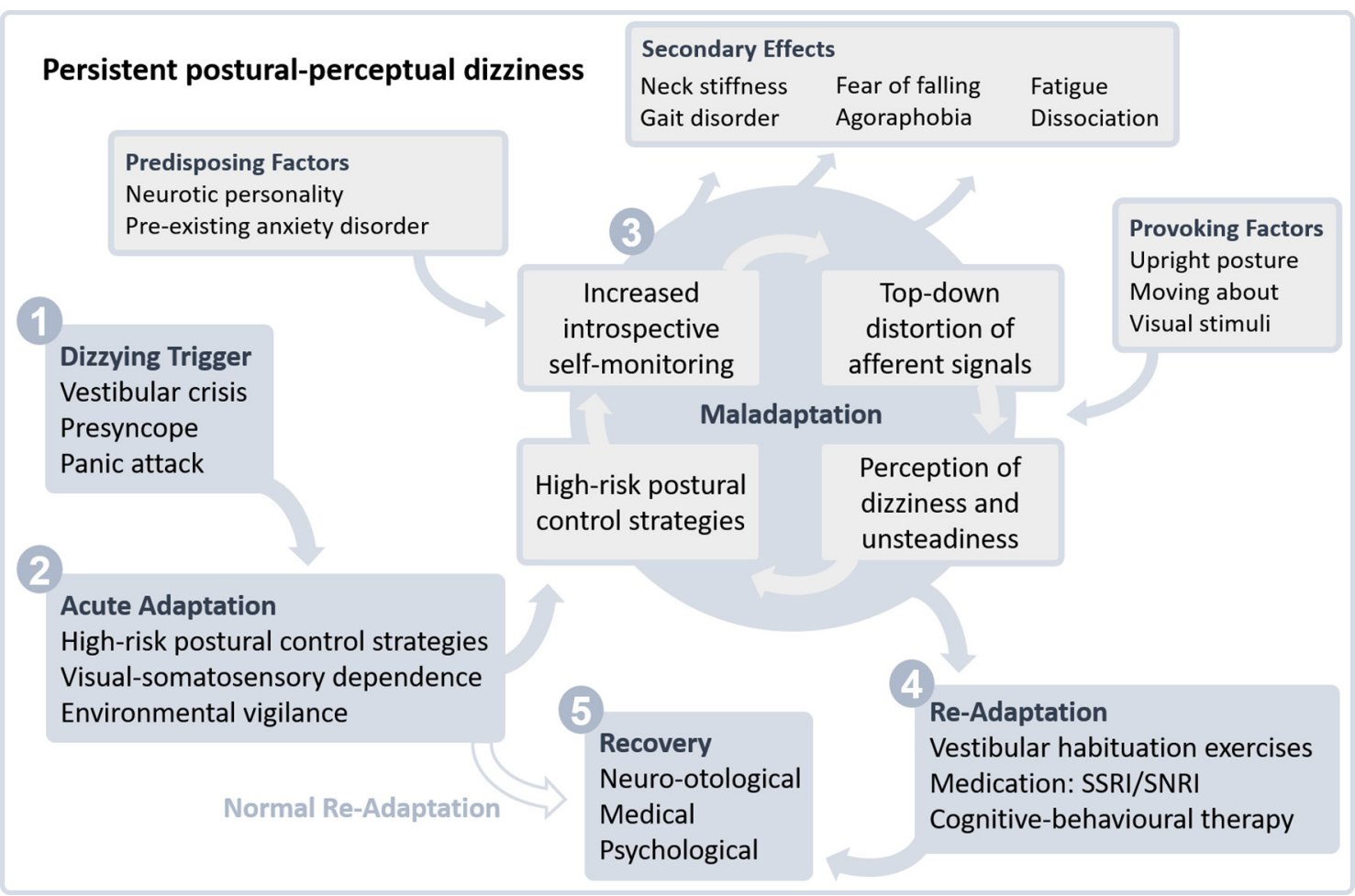

Figure 2 The normal physiological reaction to strong dizziness or dysequilibrium (1) is to activate alternative and additional systems of movement control (2) that do not rely on vestibular information. Once the acute trigger has subsided, instead of returning to normal function, a vicious cycle of maladaptation can arise (3), driven in part by excessive self-observation and anxiety. Somatosensory information about body position is thus amplified and distorted, which in turn produces subjective dizziness and leaves movement control on 'red alert'. Secondary effects like stiffening of gait, phobic avoidance and mental fatigue can develop. The aim of therapy (4) is to readapt the system to normal function by reducing anxiety and self-monitoring, habituating to provoking factors, and promoting automatic movement control until recovery (5) is achieved. SNRI, serotonin norepinephrine reuptake inhibitors; SSRI, selective serotonin reuptake inhibitors.

moving visual stimuli. In a series of studies starting in 2004 Staab and Ruckenstein ${ }^{11}$ defined chronic subjective dizziness, which overlapped significantly with phobic postural vertigo, but separated the physical symptoms from coexisting or premorbid psychopathology. PPPD was conceived in a collaborative effort among many of these researchers.

\section{PATHOPHYSIOLOGY}

Although the exact pathophysiology of PPPD remains to be elucidated, data from physiological investigations and rapidly emerging advanced structural and functional neuroimaging studies of patients with phobic postural vertigo, chronic subjective dizziness and PPPD itself have revealed three key mechanisms by which this disorder is thought to develop. ${ }^{134}$ These are stiffened postural control, a shift in processing spatial orientation information to favour visual over vestibular inputs, and failure of higher cortical mechanisms to modulate the first two processes. The normal physiological response to the onset of dizziness, vertigo or the threat of falling is to activate high-risk postural control strategies (eg, stiffened stance, shorter strides) and to rely more on visual or somatosensory inputs than vestibular signals, as can be observed in healthy individuals exposed to great heights or slippery surfaces (figure 2). ${ }^{27} 28$ Normally, these strategies are abandoned when the postural threat subsides. In patients who develop persistent dizziness following acute vestibular syndromes, however, failure of symptomatic recovery is predicted by an acute behavioural response comprising high anxiety, excessive vigilance about vestibular and balance sensations, ${ }^{29-31}$ and persistent high dependence on visual cues for spatial orientation compared with those who recover well. ${ }^{31}$ Pre-existing anxiety ${ }^{23} 32$ or neurotic personality traits ${ }^{33}$ appear to predispose patients to this problematic reaction. High-risk postural control strategies have been measured in patients with long-standing phobic postural vertigo $^{34}$ and chronic subjective dizziness, ${ }^{35}$ and persistent visual dependence has been found in patients with chronic dizziness and visual vertigo. ${ }^{31} 36$

So why is there a failure to readapt after a precipitating event? Two hypotheses have been offered. The first is based on well-established processes of Pavlovian conditioning as incorporated into cognitive-behavioural formulations of anxiety and functional disorders. Brandt and Dieterich ${ }^{25}$ suggested this for phobic postural vertigo, as did Staab $^{5}$ initially for chronic subjective dizziness. In particular, Brandt and Dieterich (see ref 4 for review) proposed that patients with phobic postural vertigo became 
consciously sensitised to transient discrepancies between predicted and actual postural movements (ie, to differences between feedforward efferent copies of planned movements and feedback afferent measurements of actual motions). Normally, these signals are used to make instinctive (ie, unconscious or preconscious) corrections of movements, but Brandt and Dieterich suggested that patients who became consciously aware of these signals exert more conscious effort into maintaining control of posture. These circumstances were thought to stand in the way of normal readaptation and, instead, set in motion a vicious cycle of maladaptation. In this model of illness, the expectation of dizziness and unsteadiness, which might be called an 'experiential engram' from the triggering episode, is awarded disproportionate predictive strength and can then distort and even over-ride bottom-up sensory input at the preconscious level of sensory integration. ${ }^{37}$ This leads to subjective sensations of dizziness during everyday vestibular and visual stimulation (eg, moving about, encountering complex visual inputs), which reinforces stiffening of stance and gait. ${ }^{15}$ The increased burden of abnormal self-monitoring could explain the common occurrence of dissociation and fatigue as results of mental overload $^{38}$; neck stiffness and functional gait disorder could develop secondary to the abnormal postural control $^{39}$; and a cycle of fear and avoidance could give rise to secondary phobic disorders such as agoraphobia. $^{23}$ Distraction from heightened attentiveness to postural control can temporarily restore healthy function $^{15}$ and can be used in therapy to facilitate functional rehabilitation. ${ }^{40}$

However, it is simplistic to assume that PPPD results solely from anxiety-driven interference with natural postural control and spatial orientation. The first neuroimaging studies of visual vertigo, ${ }^{41}$ chronic subjective dizziness ${ }^{2}$ and PPPD $^{42}$ suggest that there may be an additional pathophysiological mechanism underlying PPPD, namely a deficiency in cortical processes that subserve locomotion and spatial orientation at the highest levels. Although the research paradigms differed from one another and the use of imaging techniques for studying PPPD is just getting started, a pattern of results is beginning to take shape. Activity and connectivity appear to be greater in visual versus vestibular regions of the cortex, in keeping with physiological data on visual dependence, but activity and connectivity between key cortical regions that process space-motion information (eg, posterior insula, hippocampus) and modulate threat responses (eg, anterior cingulate cortex) are reduced. These results suggest that underactive and insufficiently interactive cortical networks may fail to suppress the bottom-up influences of instinctive threat on postural control and spatial orientation leading to sustained use of the acute high-risk strategies.

\section{TREATMENT}

\section{Communicating the diagnosis}

Treatment starts with education of the patient about the diagnosis. As with other functional disorders, some clinicians might feel the temptation to start the conversation with the last diagnostic criterion: listing negative test results and structural disorders the patient does not have. ${ }^{43}$ Instead, once the diagnosis of PPPD is suspected or ascertained, we recommend giving patients the diagnostic name and explaining that it is a well-known, common and potentially treatable cause of chronic dizziness. In our experience, it is particularly helpful to explain the nature of the disorder using a typical 'case history' (see box 2). Even if all the elements do not apply to particular patients, we usually find that it resonates well enough to give confidence that they have met a clinician who has seen this disorder before and understands it. The process of going through the typical case history leads naturally to an explanation of potential mechanisms wherein a review of figure 2 can be very informative. In particular, the figure illustrates the dynamic course of PPPD from the triggering event to persistent symptoms, highlighting the critical interplay of structural and psychological processes. This provides the background needed to introduce treatment options such as physiotherapy (to desensitise the vestibular and balance system), medications (which may alter the tone of interactions among vestibular, visual and threat systems in the brain) and psychological therapy (cognitive-behavioural 'reprogramming' to reduce heightened vigilance about dizziness and lessen worry/demoralisation about its consequences). In those patients with secondary functional gait disorder, temporary improvements of standing or walking during distraction on examination can be used to demonstrate potential reversibility to the patient. ${ }^{202143}$

In order to reinforce patient education, it is quite helpful to provide a printed fact sheet about PPPD (available at www.neurosymptoms.org/dizziness/453319731; figure 3) as well as a copy of the clinic letter to the general practitioner. This written information will offer the patient a chance to digest a diagnosis that may be hard for them to get their head round.

\section{Individualised treatment}

Having communicated the diagnosis with sufficient clarity, the next step for the consulting neurologist is to recommend appropriate therapy tailored to the needs of the patient with PPPD. For functional dizziness, as in PPPD, vestibular exercises promoting habituation are most apt. ${ }^{44} 45$ These differ from treatments used to promote recovery from structural vestibular disorders. Exercises for PPPD must be started more gently and increased more slowly. Techniques aim at fatiguing abnormal reflexive responses to movement tasks and reducing sensitivity to visual stimuli have shown longterm clinical benefit in PPPD. ${ }^{46}$ Patients can be provided with self-help material from a variety of sources (eg, 
Box 2 A typical case history of persistent postural-perceptual dizziness (PPPD) with some secondary features of anxiety that may be useful to share with a patient during explanation of the diagnosis (adapted from http:// www.neurosymptoms.org)

A 24-year-old woman gave a history of persistent dizziness for the previous 2 years. She had an initial bout of spinning vertigo, where she felt sick and could hardly get out of bed for a week. A diagnosis of vestibular neuritis was made.

\section{Core symptoms of PPPD}

As she recovered, her vestibular symptoms changed from spinning vertigo to a feeling of dizziness that she found really hard to describe. It was not the spinning sensation any more. Rather, she felt light-headed, with a sensation of swaying present mainly on standing and walking, but also noticed it when lying in bed at night if she was extremely tired. She found that she had become really sensitive to objects moving in her environment, even when she was still. It was difficult for her to use a computer or be in busy environments such as supermarkets.

\section{Secondary dissociative and anxiety symptoms}

Every now and then she also felt 'spaced out' as if she was floating and people seemed far away, which she found frightening. She thought quite often about the possibility of falling. She had only ever fallen once but had some 'near misses'. The thought of being embarrassed by falling in public made her avoid busy places. Despite her best efforts, she found that the dizziness had started to take over her life. Initially she was anxious about possible sinister diseases and found herself looking up possible causes on the internet. She then saw doctors who had carried out detailed balance tests and performed an MR scan of brain that was normal.

\section{Resolution of anxiety but persistence of PPPD with migraine}

After her medical consultation, she became more confident that she did not have a life-threatening condition. Her anxiety diminished, but she found it very hard to cope with her continuing dizziness. She developed fatigue and poor concentration and had various periods of time off work. The whole thing was made worse by regular migraines, which exacerbated her dizziness. She now felt at her wits' end.

instructional materials from the Vestibular Disorders Association are available online at http://vestibular.org/ sites/default/files/page_files/Vestibular_Rehabilitation_ $0 . p d f)$. However, patients must be instructed to start the exercises gradually and increase them slowly, but steadily. An exercise programme that is too aggressive may exacerbate symptoms, causing patients to stop far before they can achieve any benefit. Oversight of the habituation programme by an experienced vestibular therapist is recommended to ensure that it is properly designed and paced.

Selective serotonin reuptake inhibitors (SSRI) and serotonin norepinephrine reuptake inhibitors (SNRI) showed promise in reducing dizziness and unsteadiness in a majority of patients with chronic subjective dizziness, a previous incarnation of PPPD. ${ }^{5}$ Treatment with medications from these two classes of serotonergic antidepressants is supported by multiple prospective, open-label clinical trials, but no randomised controlled trials as yet. A positive response seems not to depend on the presence of psychiatric symptoms as treatment may help patients with PPPD but without psychiatric comorbidity. As expected, comorbid symptoms of depression and anxiety, where present, also improved. Dosing needs to start slowly and effects are usually seen with doses in the lower half of the ranges recommended for depression. As in many functional disorders, patients may be particularly sensitive to side effects, which led to one in five patients stopping during trials. In clinical practice, treatment is usually started with one SSRI, followed by a second SSRI trial if the first was ineffective or poorly tolerated. After that, a switch to an SNRI is recommended. Clinical response is usually seen after 8-12 weeks, and, if effective, medication should be continued for at least 1 year. Clinical experience with other classes of antidepressants has not been promising.

There is limited experience with cognitive-behavioural therapy (CBT) for treating functional dizziness. In a study on 39 patients with phobic postural vertigo, adding CBT to self-administered vestibular rehabilitation exercises had a positive short-term effect that was not sustained at 1-year follow-up. ${ }^{47}$ Newer investigations, fortunately, have been more promising. In a waiting-list controlled study on 41 patients with chronic subjective dizziness, just three sessions of CBT led to significant improvements in $75 \%$ of patients, ${ }^{49}$ which persisted at 1 and 6 months post-treatment. ${ }^{50} \mathrm{~A}$ small study on patients with functional dizziness showed that a psychotherapeutic intervention using CBT approaches led to a normalisation of postural behaviour as measured by static posturography. ${ }^{51}$ Although there is little direct evidence, CBT would be expected to help patients with pronounced fear of falling or fear of dizziness, since it can reduce similar avoidance and safety behaviour in patients with other anxiety disorders. ${ }^{40}$ In case of a secondary functional gait disorder, CBT-informed physiotherapy that focuses on illness beliefs and attention allocation can help. ${ }^{52} 53$ A recent report backed clinical experience in supporting the use of combinations of these three 
Persistent Posturo-Perceptual Dizziness

\section{(PPPD) (Functional Dizziness)}

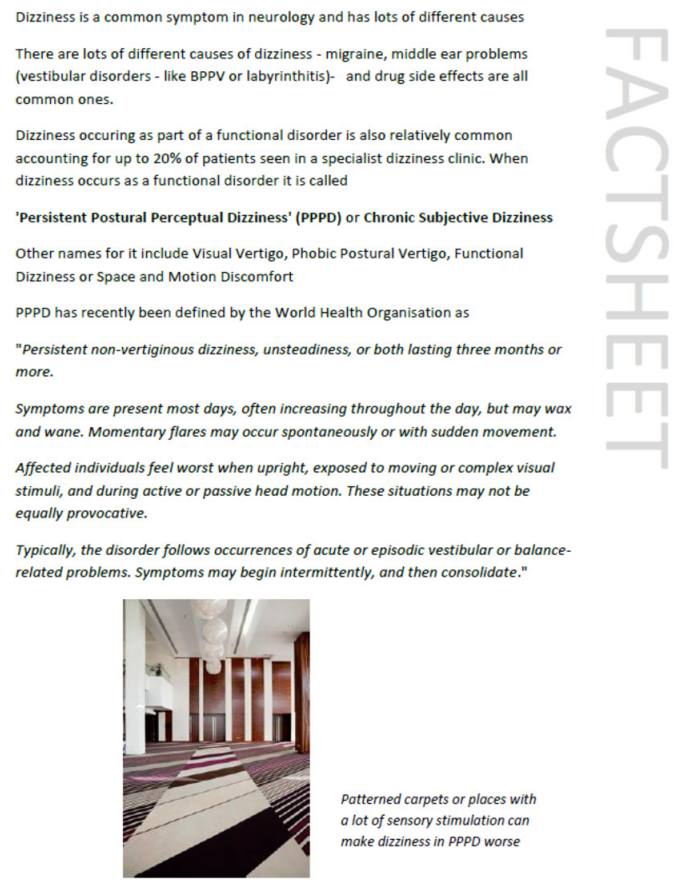

Information leaflet taken from www.neurosymptoms.org

Figure 3 Page 1 of a fact sheet available at www. neurosymptoms.org. Patients will often find such information useful to gain confidence in a diagnosis that might be unfamiliar and difficult to grasp at first.

interventions depending on patients' clinical needs and treatment preferences. ${ }^{51}$

Studies of phobic postural vertigo and chronic subjective dizziness suggest that long-term benefit probably depends on starting treatment early. ${ }^{4}$ Years of chronification usually imply a higher degree of maladaptation, more severe disability and more engrained illness

\section{Key points}

- Persistent postural-perceptual dizziness (PPPD) is a common chronic functional disorder presenting with the complaints of dizziness and unsteadiness.

- The diagnosis is made by obtaining the characteristic clinical history.

- PPPD may coexist with structural vestibular disorders, other neurological and general medical conditions, and psychiatric disorders that cause vertigo, unsteadiness or dizziness.

- Communicating the diagnosis successfully is an essential platform for further rehabilitation.

- Treatment options include vertigo rehabilitation exercises and medication, with cognitive-behavioural therapy for associated psychological morbidity, and can lead to a good outcome. beliefs. Undiagnosed or untreated patients can suffer for many years on end, which is why neurologists need to be familiar with functional dizziness and PPPD, must not shy away from a firm diagnosis and be aware of the treatment options summarised above. The presence of long duration symptoms does not preclude a good outcome with treatment.

Contributors SP wrote the first draft of the article, and all authors were involved in rewrites.

Funding This work was supported in part by a scholarship for SP from the FoRUM Forschungsreferat (research office) of the Medical Faculty of the Ruhr-University Bochum, Germany. JeS is supported by research funding from the US National Institute on Deafness and Other Communication Disorders and Mayo Clinic. JoS is supported by an NHS Scotland NRS Career Fellowship.

Competing interests None declared.

Provenance and peer review Commissioned; externally peer reviewed. This paper was reviewed by Adolfo Bronstein, London, UK.

(C) Article author(s) (or their employer(s) unless otherwise stated in the text of the article) 2018. All rights reserved. No commercial use is permitted unless otherwise expressly granted.

\section{REFERENCES}

1 Staab JP, Eckhardt-Henn A, Horii A, et al. Diagnostic criteria for persistent postural-perceptual dizziness (PPPD): consensus document of the committee for the classification of vestibular disorders of the bárány society. J Ves Res 2016.

2 Indovina I, Riccelli R, Chiarella G, et al. Role of the insula and vestibular system in patients with chronic subjective dizziness: an MRI study using sound-evoked vestibular stimulation. Front Behav Neurosci 2015;9:334.

3 Dieterich M, Staab JP. Functional dizziness: from phobic postural vertigo and chronic subjective dizziness to persistent postural-perceptual dizziness. Curr Opin Neurol 2017;30:107-13.

4 Dieterich M, Staab JP, Brandt T. Functional (psychogenic) dizziness. Handb Clin Neurol 2016;139:447-68.

5 Staab JP. Chronic subjective dizziness. Continuum 2012;18:1118-41.

6 WHO. Persistent postural-perceptual dizziness. International classification of diseases. 11th edition, 2015. http://id.who.int/ icd/entity/2005792829

7 Bisdorff AR, Staab JP, Newman-Toker DE. Overview of the international classification of vestibular disorders. Neurol Clin 2015;33:541-50.

8 Nazareth I, Yardley L, Owen N, et al. Outcome of symptoms of dizziness in a general practice community sample. Fam Pract 1999;16:616-8.

9 Stone J, Carson A, Duncan R, et al. Who is referred to neurology clinics?--the diagnoses made in 3781 new patients. Clin Neurol Neurosurg 2010;112:747-51.

10 Bronstein AM. Visual vertigo syndrome: clinical and posturography findings. J Neurol Neurosurg Psychiatry 1995;59:472-6.

11 Staab JP, Ruckenstein MJ. Expanding the differential diagnosis of chronic dizziness. Arch Otolaryngol Head Neck Surg 2007;133:170-6.

12 Spitzer RL, Kroenke K, Williams JB, et al. A brief measure for assessing generalized anxiety disorder: the GAD-7. Arch Intern Med 2006;166:1092-7. 
13 Spitzer RL, Kroenke K, Williams JBW. Validation and utility of a self-report version of PRIME-MD-The PHQ primary care study. JAMA 1999;282:1737-44.

14 Lempert T, Brandt T, Dieterich M, et al. How to identify psychogenic disorders of stance and gait.a video study in 37 patients. J Neurol 1991;238:140-6.

15 Wuehr M, Pradhan C, Novozhilov S, et al. Inadequate interaction between open- and closed-loop postural control in phobic postural vertigo. J Neurol 2013;260:1314-23.

16 Scott $\mathrm{S}$, Stone J. iPhone-responsive functional gait disorder. Pract Neurol 2014;14:422-4.

17 Stins JF, Kempe CL, Hagenaars MA, et al. Attention and postural control in patients with conversion paresis. J Psychosom Res 2015;78:249-54.

18 Wuehr M, Brandt T, Schniepp R. Distracting attention in phobic postural vertigo normalizes leg muscle activity and balance. Neurology 2017;88:284-8.

19 Querner V, Krafczyk S, Dieterich M, et al. Patients with somatoform phobic postural vertigo: the more difficult the balance task, the better the balance performance. Neurosci Lett 2000;285:21-4.

20 Stone J, Edwards M. Trick or treat? Showing patients with functional (psychogenic) motor symptoms their physical signs. Neurology 2012;79:282-4.

21 Stone J. Functional neurological disorders: the neurological assessment as treatment. Pract Neurol 2016;16:7-17.

22 Onofrj M, Thomas A, Tiraboschi P, et al. Updates on Somatoform Disorders (SFMD) in Parkinson's disease and dementia with lewy bodies and discussion of phenomenology. J Neurol Sci 2011;310:166-71.

23 Staab JP, Ruckenstein MJ. Which comes first? psychogenic dizziness versus otogenic anxiety. Laryngoscope 2003;113:1714-8.

24 Radziej K, Schmid G, Dinkel A, et al. Psychological traumatization and adverse life events in patients with organic and functional vestibular symptoms. J Psychosom Res 2015;79:123-9.

25 Brandt T, Dieterich M. Phobischer attacken schwankschwindel, ein neues syndrom? munchmed wschr. 1986:28;247-50.

26 Jacob RG, Lilienfeld SO, Furman JMR, et al. Panic disorder with vestibular dysfunction: Further clinical observations and description of space and motion phobic stimuli. J Anxiety Disord 1989;3:117-30.

27 Adkin AL, Frank JS, Carpenter MG, et al. Postural control is scaled to level of postural threat. Gait Posture 2000;12:87-93.

28 Holmberg J, Tjernström F, Karlberg M, et al. Reduced postural differences between phobic postural vertigo patients and healthy subjects during a postural threat. J Neurol 2009;256:1258-62.

29 Godemann F, Siefert K, Hantschke-Brüggemann M, et al. What accounts for vertigo one year after neuritis vestibularis - anxiety or a dysfunctional vestibular organ? J Psychiatr Res 2005;39:529-34.

30 Heinrichs N, Edler C, Eskens S, et al. Predicting continued dizziness after an acute peripheral vestibular disorder. Psychosom Med 2007:69:700-7.

31 Cousins S, Kaski D, Cutfield N, et al. Predictors of clinical recovery from vestibular neuritis: a prospective study. Ann Clin Transl Neurol 2017;4:340-6.

32 Best C, Tschan R, Eckhardt-Henn A, et al. Who is at risk for ongoing dizziness and psychological strain after a vestibular disorder? Neuroscience 2009;164:1579-87.
33 Staab JP, Rohe DE, Eggers SD, et al. Anxious, introverted personality traits in patients with chronic subjective dizziness. J Psychosom Res 2014;76:80-3.

34 Schniepp R, Wuehr M, Huth S, et al. Gait characteristics of patients with phobic postural vertigo: effects of fear of falling, attention, and visual input. J Neurol 2014;261:738-46.

35 Odman M, Maire R. Chronic subjective dizziness. Acta Otolaryngol 2008;128:1085-8.

36 Cousins S, Cutfield NJ, Kaski D, et al. Visual dependency and dizziness after vestibular neuritis. PLoS One 2014;9:e105426.

37 Edwards MJ, Adams RA, Brown H, et al. A Bayesian account of 'hysteria'. Brain 2012;135(Pt 11):3495-512.

38 Stephan KE, Manjaly ZM, Mathys CD, et al. Allostatic selfefficacy: a metacognitive theory of dyshomeostasis-induced fatigue and depression. Front Hum Neurosci 2016;10:550.

39 Pareés I, Kojovic M, Pires C, et al. Physical precipitating factors in functional movement disorders. J Neurol Sci 2014;338(1-2):174-7.

40 Whalley MG, Cane DA. A cognitive-behavioral model of persistent postural-perceptual dizziness. Cogn Behav Pract 2017;24:72-89.

41 Van Ombergen A, Heine L, Jillings S, et al. Altered functional brain connectivity in patients with visually induced dizziness. Neuroimage Clin 2017;14:538-45.

42 von Söhsten Lins EM. Functional resonance in women with persistent postural-perceptual dizziness. Brazil: Department of Otorhinolaryngology, University or São Paulo, 2015.

43 Carson A, Lehn A, Ludwig L, et al. Explaining functional disorders in the neurology clinic: a photo story. Pract Neurol 2016;16:56-61.

44 Whitney SL, Alghwiri AA, Alghadir A. An overview of vestibular rehabilitation. Handb Clin Neurol 2016;137:187-205.

45 Staab JP. Behavioral aspects of vestibular rehabilitation. NeuroRehabilitation 2011;29:183.

46 Thompson KJ, Goetting JC, Staab JP, et al. Retrospective review and telephone follow-up to evaluate a physical therapy protocol for treating persistent postural-perceptual dizziness:a pilot study. J Vestib Res 2015;25:97-103.

47 Holmberg J, Karlberg M, Harlacher U, et al. Treatment of phobic postural vertigo. A controlled study of cognitivebehavioral therapy and self-controlled desensitization. J Neurol 2006;253:500-6.

48 Holmberg J, Karlberg M, Harlacher U, et al. One-year followup of cognitive behavioral therapy for phobic postural vertigo. J Neurol 2007;254:1189-92.

49 Edelman S, Mahoney AE, Cremer PD. Cognitive behavior therapy for chronic subjective dizziness: a randomized, controlled trial. Am J Otolaryngol 2012;33:395-401.

50 E J Mahoney A, Edelman S, D Cremer P. Cognitive behavior therapy for chronic subjective dizziness: longer-term gains and predictors of disability. Am J Otolaryngol 2013;34:115-20.

51 Best C, Tschan R, Stieber N, et al. STEADFAST: psychotherapeutic intervention improves postural strategy of somatoform vertigo and dizziness. Behav Neurol 2015;2015:1-10.

52 Nielsen G, Stone J, Matthews A, et al. Physiotherapy for functional motor disorders: a consensus recommendation. J Neurol Neurosurg Psychiatry 2015;86:1113-9.

53 Nielsen G, Buszewicz M, Stevenson F, et al. Randomised feasibility study of physiotherapy for patients with functional motor symptoms. J Neurol Neurosurg Psychiatry 2017;88:484-90. 